\title{
Establishing quantum gravity using Nanotechnology
}

\author{
Zaid $\mathrm{Zaz}^{1 *}$ and Anha Bhat ${ }^{2}$ \\ Department of Electronics and Communication Engineering, University of Kashmir, Srinagar, \\ Kashmir, 190006, India \\ Department of Physics, National Institute of Technology, Srinagar, Kashmir, 190006, India \\ *Email: mohammadzaz@gmail.com
}

Experimentally verifying the theories of quantum gravity has been quite a challenge in modern theoretical physics. Recently many investigations have signaled the existence of a minimal measurable length and a maximum measurable momentum [1]. This observation is consistent with modern theories of quantum gravity such as String theory, Loop quantum gravity and doubly special relativity. It has been seen that the existence of a minimal length and consequently a maximum measurable momentum modifies the Heisenberg's uncertainty principle $\Delta x \cdot \Delta y \geq \frac{\hbar}{2}$ a so-called generalized uncertainty principle (GUP).

$$
\begin{gathered}
\Delta x . \Delta y \geq \frac{\hbar}{2}\left[1-2 \alpha\langle p\rangle+4 \alpha^{2}\left((\Delta p)^{2}\right.\right. \\
\left.\left.+\langle p\rangle^{2}\right)\right]
\end{gathered}
$$

Where $\alpha=\frac{\alpha_{0}}{M_{p C}}=\frac{\alpha_{0}}{\hbar} l$,

Also, $M_{p}=$ Planck mass, $l_{p}=$ Planck length $\approx$ $10^{-35} \mathrm{~m}$ and $\alpha_{0}$ is a constant of the order of unity. This condition is imposed due to various physical motivations however this condition need not be imposed in anticipation [2].

This is known to modify all the known quantum mechanical Hamiltonians at the Planck scale. GUP corrections to various quantum mechanical phenomena have been calculated and it has been seen that the implications of these corrections may be experimentally verifiable [2].

In this work our focus shall be on the GUP induced modifications to the phenomenon of quantum mechanical tunneling. We shall review the GUP induced modifications to tunneling in a scanning tunneling microscope and it shall be seen that a deviation in tunneling current is obtained which might be measurable in an optimistic scenario. The same formalism shall then be applied to a tunnel diode and a modified tunneling current shall be obtained, the same general result shall be particularized to Nano tunnel elements such as Si-Ge heterojunctions. SEM image of a Ge nanowire and a schematic of the cross section of a vertical tunnel diode has been shown in Figure 1 [3]. A comparison between modified tunneling currents arising due to different bounds used for $\alpha_{0}$ calculated using GUP modifications to other physical phenomenon shall be made. We shall also attempt to calculate a new bound for $\alpha_{0}$ using Si-Ge heterojunction.
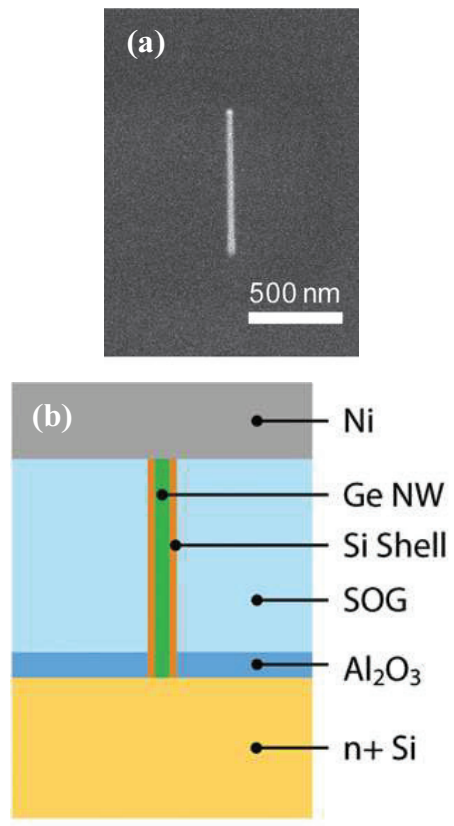

Figure 1: (a) SEM image of a Ge nanowire and (b) schematic of the cross section of a vertical tunnel diode. Images adopted form [3]

\section{References}

1. Hossenfleder, S Minimal length scenarios for quantum gravity.[arxiv:1203.6191],86

2. Ahmed Farag Ali, Surya Das, Elias C. Vagenas A proposal for testing quantum gravity in lab [arxiv:1107.31642]

3. W.Y. Fung, L. Chen, W. Lu, "Esaki tunnel diodes based on vertical Si-Ge nanowire heterojunctions," Appl. Phys. Lett., 99[9] 092108(2011). 